Journal of Patient-Centered

\title{
Heart-Healthy Integrative Nutritional Counseling Group Education Sessions Among Chinese Americans With Cardiovascular Risk Factors or Disease: A Primary Care Quality Improvement Pilot
}

Jasmin Woo

Evelyn Y. Ho

Jane Jih

Follow this and additional works at: https://aah.org/jpcrr

Part of the Alternative and Complementary Medicine Commons, Cardiology Commons, Cardiovascular Diseases Commons, Community Health and Preventive Medicine Commons, Dietetics and Clinical Nutrition Commons, Integrative Medicine Commons, Internal Medicine Commons, and the Primary Care Commons

\section{Recommended Citation}

Woo J, Ho EY, Jih J. Heart-healthy integrative nutritional counseling group education sessions among Chinese Americans with cardiovascular risk factors or disease: a primary care quality improvement pilot. J Patient Cent Res Rev. 2021;8:127-33. doi: 10.17294/2330-0698.1783

Published quarterly by Midwest-based health system Advocate Aurora Health and indexed in PubMed Central, the Journal of Patient-Centered Research and Reviews (JPCRR) is an open access, peer-reviewed medical journal focused on disseminating scholarly works devoted to improving patient-centered care practices, health outcomes, and the patient experience. 


\title{
Heart-Healthy Integrative Nutritional Counseling Group Education Sessions Among Chinese Americans With Cardiovascular Risk Factors or Disease: A Primary Care Quality Improvement Pilot
}

\author{
Jasmin Woo, BS, ${ }^{1,2}$ Evelyn Y. Ho, PhD, ${ }^{3,4}$ Jane Jih, MD, MPH, MAS ${ }^{1,4,5}$ \\ ${ }^{1}$ Division of General Internal Medicine, University of California, San Francisco, San Francisco, CA; ${ }^{2}$ School of Nursing, \\ University of California, San Francisco, San Francisco, CA; ${ }^{3}$ Department of Communication Studies, University of San \\ Francisco, San Francisco, CA; ${ }^{4}$ Asian American Research Center on Health, San Francisco, CA; ${ }^{5}$ Multiethnic Health \\ Equity Research Center, University of California, San Francisco, San Francisco, CA
}
Purpose Current biomedical cardiovascular disease nutrition counseling does not incorporate Chinese medicine principles.

Methods A heart-healthy integrative nutritional counseling (H2INC) curriculum consistent with Chinese medicine principles and biomedical nutrition guidelines was taught to Chinese Americans in group education sessions. Chinese-speaking patients with cardiovascular disease or risk factors from an urban general medicine practice were recruited to attend a 90-minute group session. Participants completed prepost surveys to assess the impact of $\mathrm{H} 2 \mathrm{INC}$ on their perceived heart-healthy nutrition knowledge and empowerment, as well as the cultural relevance of $\mathrm{H} 2 \mathrm{INC}$.

Results A total of 47 participants (mean age: 74 years; $63.8 \%$ female) attended a session. In response to the statement "I am able to choose heart-healthy Chinese foods to eat," on a 5-point Likert scale for which "strongly disagree" $=1$ and "strongly agree" $=5$, the presession survey mean response was $3.87 \pm 0.69$ and the postsession survey mean response was $4.13 \pm 0.58(P=0.05)$. Postsession, $87 \%$ of participants agreed or strongly agreed that they felt confident using what they learned and $94 \%$ agreed or strongly agreed that H2INC fit their culture.

Conclusions H2INC had a positive impact on perceived heart-healthy nutrition knowledge and empowerment and rated high in cultural relevance. Culturally relevant education sessions like H2INC could be a promising primary care health education intervention. (J Patient Cent Res Rev. 2021;8:127-133.)

Keywords Chinese Americans; cardiovascular health; integrative medicine; nutrition counseling; primary care; culturally tailored education

$\mathrm{A}$ sian Americans are a growing racial/ethnic group in the United States, with Chinese being the largest subgroup; among Chinese Americans, $63 \%$ are immigrants and $41 \%$ have limited English proficiency. ${ }^{1}$ Cardiovascular disease (CVD) is a largely unaddressed yet salient health issue among Chinese Americans. One reason could be because of the model minority myth, ${ }^{2,3}$ which falsely identifies Asian Americans as being free from illnesses such as obesity. While other communities of color demonstrate improvements in CVD

Corresponding author: Jane Jih, MD, MPH, MAS, UCSF Division of General Internal Medicine, 1545 Divisadero Street, San Francisco, CA 94115 (jane.jih@ucsf.edu) mortality, the same cannot be said for Asian Americans. ${ }^{4}$ From a population-based study of U.S. adults without clinical CVD, the percentage of treated but uncontrolled hypertension (a risk factor for CVD) was 33\% for Chinese Americans and significantly higher compared to Whites. ${ }^{5}$ Greater fruit and vegetable consumption is associated with a lower risk of stroke and myocardial infarction, ${ }^{6}$ but Chinese Americans eat fewer vegetables and fruits than recommended, ${ }^{7}$ and less than half adhere to low-salt diets. ${ }^{8}$ Thus, Chinese Americans are at increased risk of CVD. ${ }^{9,10}$

Often times Chinese Americans use Chinese medicine principles, such as balancing the hot and cold properties of foods ${ }^{11}$ or ingesting tonic soups, to self-manage CVD. ${ }^{11,12}$ For Chinese immigrants, dietary acculturation is a multidimensional process in which individuals often 
find various ways to use and retain traditional foods and incorporate nontraditional foods. ${ }^{13}$ Current biomedical CVD nutrition guidelines do not take into account these or other Chinese medicine principles. Western nutritional tools like MyPlate ${ }^{14}$ focus on a broad variety of food groups for individual meals, whereas in Chinese dining culture, dishes are often shared family style. ${ }^{15}$

Diverse populations often require targeted efforts to understand particular disparities and needs. ${ }^{16} \mathrm{~A}$ ChineseEnglish guidebook and patient education program titled Heart-Healthy Integrative Nutrition Counseling (H2INC) - which blended Chinese medicine dietary principles (eg, hot/cold and seasonal foods) and biomedical nutritional guidelines (eg, avoiding excess salt, fat, and sugars) - was created through an iterative multidisciplinary process. ${ }^{17}$ This paper describes a pilot quality improvement project of group education teaching of H2INC to Chinese Americans with cardiovascular risk factors and/or CVD receiving care from the University of California, San Francisco (UCSF) general internal medicine practice. It also reports the impact of H2INC on participants' perceived heart-healthy nutrition knowledge and empowerment and the cultural relevance of H2INC.

\section{METHODS}

\section{Study Design}

The study period was June 2018 to August 2018. The objective was to enhance the quality of care provided to adult Chinese American patients in UCSF's general internal medicine practice. This project was approved by faculty practice leadership and met the definition of a quality improvement project at UCSF; thus, institutional review board review was not required.

\section{Creation of H2INC Guide}

In our prior work, we created a H2INC ChineseEnglish guidebook consistent with current biomedical nutrition concepts and Chinese medicine principles. ${ }^{17}$ The H2INC guidebook was created in 3 phases. Phase 1 included semi-structured individual interviews with Chinese medicine practitioners and lay experts. Phase 2 included semi-structured individual interviews with primary care clinicians, CVD-related specialists, and dietitians, followed by interviews with licensed Chinese medicine experts for a consensus draft of the guide. Phase 3 included focus groups with 20 Chinese American patients from the study site who self-reported at least one cardiovascular risk factor and/or CVD. The guide can be accessed at www.INCguide.org.

\section{Recruitment}

We used the electronic health record to identify patients that met the following criteria: 1) age 18 years or older;
2) Chinese-speaking (Cantonese or Mandarin); and 3) at least one cardiovascular risk factor and/or CVD among hypertension, dyslipidemia, coronary artery disease, cerebrovascular disease, and congestive heart failure, per International Classification of Diseases, Tenth Revision (ICD-10) diagnosis codes.

A subset of eligible patients were randomly selected to receive a 1-page Chinese-English flyer invitation by mail to attend a free 90-minute in-person group education session in Cantonese or Mandarin to learn about heart health using an integrative diet with Chinese principles and Western medicine concepts. We mailed 200 invitations to Cantonese-speaking patients and 75 to Mandarin-speaking patients. All patients from Phase 3 of the H2INC development were invited to attend. Interested patients called the language-concordant project team to sign up to attend.

\section{Education Session Procedures}

The H2INC guide served as the basis for the group education session curriculum. Sessions were held in a conference room co-located within the practice and were led by a bilingual (English/Cantonese or English/ Mandarin) lay health educator or community Chinese medicine expert, both of whom received brief training of the H2INC curriculum through iterative discussions with the investigators who developed H2INC. The total number of group education sessions offered was based on the number and language preferences of patients who expressed interest in attending. Participants were invited to independently complete a presession survey in their preferred language and then received a full-color Chinese-English H2INC guide to follow along with the health educator. Each session lasted about 90 minutes. At the end of the session, participants were invited to complete a postsession survey and offered an additional H2INC guide to share with family or friends.

\section{Measures}

Participants completed pre- and postsession surveys in Chinese. The presession survey collected participant demographics (age, gender) and self-reported cardiovascular risk (selecting all relevant conditions from a list that included hypertension, dyslipidemia, congestive heart failure, stroke, heart rhythm problems, heart disease including coronary artery disease or myocardial infarction, other [fill in], or none).

As presented in Table 1, the presession survey assessed baseline perceived heart-healthy nutrition knowledge (3 questions) and a baseline measure of empowerment (1 question). The postsession survey reassessed these measures and assessed 2 additional measures 
Table 1. Participant Survey Questions/Statements and Answer Choices for Perceived Heart-Healthy Nutrition Knowledge and Empowerment, Cultural Relevance of the Heart-Healthy Integrative Nutritional Counseling, and Importance of Receiving Heart-Health Information

\begin{tabular}{|c|c|c|}
\hline Measures & Survey Questions/Statements & Answer Choices \\
\hline $\begin{array}{l}\text { Perceived heart-healthy } \\
\text { nutrition knowledge } \\
\text { (presession and postsession } \\
\text { surveys) }\end{array}$ & $\begin{array}{l}\text { "I know what to eat and what to avoid to keep my heart } \\
\text { healthy." } \\
\text { "Eating healthy foods is beneficial for my heart health." } \\
\text { "I am able to choose heart-healthy Chinese foods to eat." } \\
\text { "I feel confident that I can stick with eating healthy foods." }\end{array}$ & $\begin{array}{l}5 \text {-point Likert scale: } \\
1=\text { Strongly Disagree } \\
2=\text { Disagree } \\
3=\text { Neutral } \\
4=\text { Agree }\end{array}$ \\
\hline $\begin{array}{l}\text { Participant empowerment } \\
\text { (postsession survey only, } \\
\text { except where noted) }\end{array}$ & $\begin{array}{l}\text { "I feel confident that I can stick with eating healthy foods."* } \\
\text { "I feel confident that I will use the things I learned today." } \\
\text { "In my next meal, I plan to use the things I learned today." }\end{array}$ & $5=$ Strongly Agree \\
\hline $\begin{array}{l}\text { Cultural relevance } \\
\text { (postsession survey only) }\end{array}$ & "The diet I learned today fit my culture." & \\
\hline $\begin{array}{l}\text { Sharing information learned } \\
\text { (postsession survey only) }\end{array}$ & $\begin{array}{l}\text { "I plan to share details of what I learned today with a } \\
\text { friend." } \\
\text { "I plan to share details of what I learned today with a } \\
\text { family member." } \\
\text { "I plan to share details of what I learned today with my } \\
\text { doctor or nutritionist." } \\
\text { "I plan to share details of what I learned today with any } \\
\text { other health provider." }\end{array}$ & \\
\hline $\begin{array}{l}\text { Importance of heart-health } \\
\text { information sources } \\
\text { (presession survey only) }\end{array}$ & $\begin{array}{l}\text { Health education programs (like this one) } \\
\text { Friends and family } \\
\text { Through the internet } \\
\text { Doctors } \\
\text { Nurses/Nurse practitioners } \\
\text { Traditional Chinese medicine practitioners } \\
\text { Nutritionist/Dietician } \\
\text { Newspapers/Magazines } \\
\text { Books } \\
\text { Radio } \\
\text { Television }\end{array}$ & $\begin{array}{l}\text { 5-point Likert scale: } \\
1 \text { = Not Important } \\
2 \\
3 \text { = Somewhat Important } \\
4 \\
5 \text { = Definitely Important }\end{array}$ \\
\hline
\end{tabular}

*This statement was asked in both the presession and postsession surveys.

of empowerment and a cultural relevance measure. Additional survey questions and the 5-point Likert scales used for participant responses to survey statements are described in Table 1.

\section{Analysis}

Univariate analyses were conducted using Research Electronic Data Capture (REDCap) and Microsoft Excel software. Unpaired $t$-tests with a two-tailed hypothesis were conducted to compare the mean responses of survey questions assessed in both the preand postsession surveys, using SPSS Statistics software (IBM Corporation). A P-value of $<0.05$ was used as the threshold for measuring statistical significance.

\section{RESULTS}

Three one-time group education sessions were offered 2 in Cantonese and 1 in Mandarin. A total of 24 Chinese Americans attended the 2 Cantonese-language sessions, and 23 Chinese Americans attended the Mandarin- 
Table 2. Self-Reported Sociodemographic and Clinical Characteristics of Chinese American Participants ( $N=47)$ Attending an H2INC Group Education Session

\begin{tabular}{lc}
\hline & $\begin{array}{c}\text { Percentage or } \\
\text { Characteristic }\end{array}$ \\
\hline Female sex & $63.8 \%$ \\
Mean age in years & $74 \pm 10(51-90)$ \\
Self-reported cardiovascular risk factor and/or disease* & $54.5 \%$ \\
Hypertension & $54.5 \%$ \\
Dyslipidemia & $13.6 \%$ \\
Coronary heart disease/coronary artery disease including myocardial infarction & $11.4 \%$ \\
Heart rhythm problems & $9.1 \%$ \\
Stroke & $2.3 \%$ \\
Congestive heart failure & $15.9 \%$ \\
Other (atrial fibrillation, fatigue, hypotension, pleural effusion, cardiac stent, prediabetes, & $13.6 \%$ \\
$\quad$ rheumatoid arthritis) & \\
None reported & \\
\hline
\end{tabular}

*Participants could report more than one category.

H2INC, heart-healthy integrative nutritional counseling; SD, standard deviation.

Table 3. Mean Survey Response Scores* for Perceived Heart-Healthy Nutrition Knowledge and Empowerment Among Chinese American Participants Attending an H2INC Group Education Session

\begin{tabular}{|c|c|c|c|}
\hline Survey Question & $\begin{array}{c}\text { Presession, } \\
\text { Mean } \pm \text { SD }\end{array}$ & $\begin{array}{c}\text { Postsession, } \\
\text { Mean } \pm \text { SD }\end{array}$ & $\boldsymbol{P}^{\dagger}$ \\
\hline \multicolumn{4}{|l|}{ Perceived Heart-Healthy Nutrition Knowledge } \\
\hline "I know what to eat and what to avoid to keep my heart healthy." & $3.95 \pm 0.62$ & $4.13 \pm 0.72$ & 0.21 \\
\hline "Eating healthy foods is beneficial for my heart health." & $4.22 \pm 0.55$ & $4.39 \pm 0.54$ & 0.13 \\
\hline "I am able to choose heart healthy Chinese foods to eat." & $3.87 \pm 0.69$ & $4.13 \pm 0.58$ & 0.05 \\
\hline \multicolumn{4}{|l|}{ Participant Empowerment } \\
\hline "I feel confident that I can stick with eating healthy foods." & $3.87 \pm 0.63$ & $4.09 \pm 0.63$ & 0.10 \\
\hline
\end{tabular}

${ }^{*}$ Participants were not required to answer every survey question. For Table 3 questions, 46 of 47 participants registered answers on a 5-point Likert scale (1 = Strongly Agree, $2=$ Disagree, $3=$ Neutral, $4=$ Agree, and $5=$ Strongly Agree) .

${ }^{\dagger} P$-values were calculated from an unpaired t-test comparing presession and postsession survey means for each survey question. A P-value of $<0.05$ was used as the threshold for measuring statistical significance.

H2INC, heart-healthy integrative nutritional counseling; SD, standard deviation.

language session. Mean age of all 47 participants was $74 \pm 10$ years; $63.8 \%$ were female. Hypertension and dyslipidemia ( $54.5 \%$ each) were the most prevalent selfreported cardiovascular risk factors or CVD (Table 2).

\section{Survey Measures}

Perceived Heart Health Nutrition Knowledge. Pre- and postsession survey measures of participants' perceived heart-healthy nutrition knowledge showed improvements after the education (Table 3). In response to the statement
"I am able to choose heart-healthy Chinese foods to eat," the mean Likert response in the postsession survey was $4.13 \pm 0.58$, a notable increase from a mean of $3.87 \pm 0.69$ in the presession survey $(\mathrm{P}=0.05)$.

Participant Empowerment. In response to the statement, "I feel confident that I can stick with eating healthy foods," the mean response in the postsession survey was $4.09 \pm 0.63$, a nonsignificant increase from a mean of $3.87 \pm 0.63$ in the presession survey $(\mathrm{P}=0.10)$ (Table 3 ). 
In the postsession survey, $86.9 \%$ of participants agreed or strongly agreed that they felt confident that they would use the things they learned in the group education session, and $91.3 \%$ of participants agreed or strongly agreed that they plan to use the things they learned today in their next meal (Table 4).

Cultural Relevance. In the postsession survey, $93.6 \%$ of participants agreed or strongly agreed that the diet they learned in the education session fit their culture (Table 4).

\section{Additional H2INC Measures}

Participants also reported that they agreed or strongly agreed with statements to share details of what they learned in the group education session with a family member $(95.7 \%)$, friend $(89.1 \%)$, or health care provider other than their doctor $(88.9 \%)$, followed by their doctor or nutritionist (84.4\%). In participants' ratings of importance of heart-health information sources (Table 5),

Table 4. Empowerment and Cultural Relevance Measures for the H2INC Group Education Session Among Postsession Survey Respondents $(\mathrm{N}=47)$

\begin{tabular}{lc}
\hline Measure & Percentage \\
\hline Additional Participant Empowerment & \\
"I feel confident that I will use the things I & \\
learned today." & \\
Strongly Agree & $23.9 \%$ \\
Agree & $63.0 \%$ \\
Neutral & $10.9 \%$ \\
Disagree & $2.2 \%$ \\
Strongly Disagree & -- \\
"In my next meal, I plan to use the things I & \\
learned today." & \\
Strongly Agree & $23.9 \%$ \\
Agree & $67.4 \%$ \\
Neutral & $8.7 \%$ \\
Disagree & -- \\
Strongly Disagree & -- \\
Cultural Relevance Measure & \\
"The diet I learned today fits my culture." & \\
Strongly Agree & \\
Agree & \\
Neutral & \\
Disagree & -- \\
Strongly Disagree & $-17.0 \%$ \\
\hline
\end{tabular}

H2INC, heart-healthy integrative nutritional counseling. a health education program like the one presented in this study (4.44 \pm 0.81$)$ was rated as equally as important as doctors $(4.44 \pm 0.78)$.

\section{DISCUSSION}

In this pilot quality improvement project at an academic primary care practice, we found that H2INC showed promise as a quick, one-session health education tool. Similar to other studies showing favorable increases in nutrition knowledge and empowerment among older Asian Americans attending nutrition-focused educational sessions, ${ }^{7,18}$ our findings suggest that the H2INC group education sessions had an overall positive impact on participants' perceived heart-healthy nutrition knowledge and empowerment and were rated as highly culturally relevant.

Numerous studies have reported that physicians are a primary and trusted source for health information for Chinese Americans. ${ }^{19-21}$ However, if health programs such as $\mathrm{H} 2 \mathrm{INC}$ can be rated as highly as physicians as source for health information, this suggests that it may be worthwhile to invest in culturally and linguistically appropriate health education programs that recognize and acknowledge the culturally relevant health practices of patients within clinical settings. Participants may have reported a positive impact of the group education session based, in part, on

Table 5. Mean Ratings* Among Chinese Americans Who Attended H2INC Regarding Importance of Receiving Heart-Health Information, by Source

\begin{tabular}{ll}
\hline Heart-Health Information Source & Mean \pm SD \\
\hline Health education programs (like this one) & $4.44 \pm 0.81$ \\
Doctors & $4.44 \pm 0.78$ \\
Nurse/Nurse practitioners & $4.19 \pm 0.89$ \\
Nutritionist/Dietician & $4.10 \pm 0.97$ \\
Traditional Chinese medicine practitioners & $3.93 \pm 1.21$ \\
Friends and family & $3.93 \pm 0.96$ \\
Television & $3.52 \pm 1.00$ \\
Through the internet & $3.50 \pm 1.26$ \\
Books & $3.47 \pm 1.10$ \\
Newspapers/Magazines & $3.40 \pm 1.18$ \\
Radio & $3.15 \pm 1.23$ \\
\hline
\end{tabular}

*45 of 47 survey participants answered this question on a 5-point Likert scale ranging from Not Important (1) to Somewhat Important (3) to Definitely Important (5).

$H 2 I N C$, heart healthy integrative nutritional counseling; SD, standard deviation. 
the tailored nature of the H2INC curriculum, as H2INC integrates relevant traditional cultural beliefs and eating habits while encouraging a balance of healthy dietary practices promoted within national dietary guidelines.

In addition to the 3 one-time $\mathrm{H} 2 \mathrm{INC}$ group education sessions, we co-hosted an interactive community-based H2INC group education session with our co-institutional Asian Health Institute, an academic-based center that offers health education programs to the San Francisco Bay Area Asian American community. A total of 74 community participants attended that session to learn about H2INC, underscoring the need and demand for culturally and linguistically relevant health education programs. With limited existing interventions to address the heart-health education needs of Chinese Americans with cardiovascular risk factors and/or CVD, H2INC could be a valuable patient-centered tool to address this shortcoming in both clinical and community settings.

\section{Limitations}

This study had several limitations. First, a small sample size limited our ability to assess differences in pre- and postsession survey measures. Second, ascertainment bias cannot be excluded. Third, we measured participantperceived heart-health nutrition knowledge and did not assess the impact of the one-time group education session on self-reported patient behaviors and clinical outcomes at a later date; however, this is an important direction for future research. Lastly, our findings may not be fully generalizable to the general Chinese American population because participants were recruited from a convenience sample from an academic primary care setting in one geographical location.

The methodological strengths of this study include inlanguage group education sessions conducted within the general medicine practice, teaching of the group education session through oral discussion and colorprinted booklets for participants to follow along during session and to take home, and culturally tailored education for this underserved population.

\section{CONCLUSIONS}

With a lack of integrative in-language health information sources for the Chinese American population, it is important that culturally and linguistically appropriate health education programs, such as heart-healthy integrative nutritional counseling, be accessible to promote health equity. Future research should focus on the impact of H2INC on clinical and health care utilization outcomes and evaluate which aspects of H2INC are most important to promote sustained behavior change for heart health.

\section{Patient-Friendly Recap}

- Cardiovascular disease is an important health issue among Chinese Americans, but the heart-based nutritional counseling provided in clinics is often not culturally relevant or linguistically suitable.

- The authors initiated group education sessions on heart-healthy nutrition that were designed for Chinese American patients at a primary care practice in San Francisco, California.

- Self-reported knowledge of heart-healthy Chinese foods was modestly improved after the education, and the nutritional counseling curriculum was well received as useful and culturally relevant.

\section{Acknowledgments}

We would like to thank the participants for sharing their time and knowledge with us as well as research assistants Choi Yu, Stephanie Wong, Annalise Stromsta, Ryan Qiu, and Ashley Liu and graphic designers Ashley Lin, Ema Matsumura, Yvonne Chow, and Antony Nguyen.

\section{Author Contributions}

Study design: all authors. Data acquisition or analysis: all authors. Manuscript drafting: all authors. Critical revision: all authors.

\section{Conflicts of Interest}

None.

\section{Funding Sources}

This work was supported by funding from the Mount Zion Health Fund (grant no. 20160995), the University of San Francisco Faculty Development Fund, and the National Center for Advancing Translational Sciences of the National Institute of Health (award no. KL2TR001870). The funding sources had no role or involvement in the design and conduct of the study; the collection, management, analysis, or interpretation of the data; or in the preparation, review, or approval of the manuscript. Contents are solely the responsibility of the authors and do not necessarily represent the official views of the funders.

\section{References}

1. Pew Research Center's Social \& Demographic Trends. Chinese in the U.S. fact sheet. Published September 8, 2017; accessed April 28, 2020. https://www.pewsocialtrends.org/fact-sheet/ asian-americans-chinese-in-the-u-s/

2. Yi SS, Kwon SC, Sacks R, Trinh-Shevrin C. Commentary: Persistence and health-related consequences of the model minority stereotype for Asian Americans. Ethn Dis. 2016;26:133-8. $\underline{\text { CrossRef }}$

3. Lee $\mathrm{S}$, Martinez $\mathrm{G}, \mathrm{Ma} \mathrm{G}$, et al. Barriers to health care access in 13 Asian American communities. Am J Health Behav. 2010;34:21-30. CrossRef

4. Jose PO, Frank ATH, Kapphahn KI, et al. Cardiovascular disease mortality in Asian Americans. $\mathrm{J} \mathrm{Am} \mathrm{Coll} \mathrm{Cardiol.}$ 2014;64:2486-94. $\underline{\text { CrossRef }}$ 
5. Kramer H, Han C, Post W, et al. Racial/ethnic differences in hypertension and hypertension treatment and control in the multi-ethnic study of atherosclerosis (MESA). Am J Hypertens. 2004;17:963-70. CrossRef

6. McGuire S. Reports from the agencies. U.S. Department of Agriculture and U.S. Department of Health and Human Services, Dietary Guidelines for Americans, 2010. 7th Edition, Washington, DC: U.S. Government Printing Office, January 2011. Adv Nutr. 2011;2:293-4. CrossRef

7. Jih J, Le G, Woo K, et al. Educational interventions to promote healthy nutrition and physical activity among older Chinese Americans: a cluster-randomized trial. Am J Public Health. 2016;106:1092-8. CrossRef

8. Firestone MJ, Beasley JM, Kwon SC, Ahn J, Trinh-Shevrin C, Yi SS. Asian American dietary sources of sodium and salt behaviors compared with other racial/ethnic groups, NHANES, 2011-2012. Ethn Dis. 2017;27:241-8. CrossRef

9. Palaniappan LP, Araneta MRG, Assimes TL, et al. Call to action: cardiovascular disease in Asian Americans: a science advisory from the American Heart Association. Circulation. 2010;122:1242-52. CrossRef

10. Palaniappan L, Wang Y, Fortmann SP. Coronary heart disease mortality for six ethnic groups in California, 1990-2000. Ann Epidemiol. 2004;14:499-506. CrossRef

11. Jiang S, Quave CL. A comparison of traditional food and health strategies among Taiwanese and Chinese immigrants in Atlanta, Georgia, USA. J Ethnobiol Ethnomed. 2013;9(1):61. CrossRef

12. Rong X, Peng Y, Yu HP, Li D. Cultural factors influencing dietary and fluid restriction behaviour: perceptions of older Chinese patients with heart failure. J Clin Nurs. 2017;26:717-26. CrossRef

13. Deng F, Zhang A, Chan CB. Acculturation, dietary acceptability, and diabetes management among Chinese in North America. Front Endocrinol (Lausanne). 2013;4:108. CrossRef
14. U.S. Department of Agriculture. What is MyPlate? Accessed August 28, 2020. https://www.choosemyplate.gov/ WhatIsMyPlate

15. Ma G. Food, eating behavior, and culture in Chinese society. $J$ Ethn Foods. 2015;2:195-9. CrossRef

16. Satia JA, Patterson RE, Taylor VM, et al. Use of qualitative methods to study diet, acculturation, and health in ChineseAmerican women. J Am Diet Assoc. 2000;100:934-40. CrossRef

17. Ho EY, Acquah J, Chao C, et al. Heart healthy integrative nutritional counseling (H2INC): Creating a Chinese medicine + western medicine patient education curriculum for Chinese Americans. Patient Educ Couns. 2018;101:2202-8. CrossRef

18. Jih J, Stewart SL, Luong TN, Nguyen TT, McPhee SJ, Nguyen $\mathrm{BH}$. A cluster randomized controlled trial of a lay health worker intervention to increase healthy eating and physical activity among Vietnamese Americans. Prev Chronic Dis. 2020;17:E33. CrossRef

19. Meissner HI, Potosky AL, Convissor R. How sources of health information relate to knowledge and use of cancer screening exams. J Community Health. 1992;17:153-65. CrossRef

20. Hesse BW, Nelson DE, Kreps GL, et al. Trust and sources of health information: the impact of the internet and its implications for health care providers: findings from the first Health Information National Trends Survey. Arch Intern Med. 2005;165:2618-24. CrossRef

21. O'Malley AS, Kerner JF, Johnson L. Are we getting the message out to all? Health information sources and ethnicity. Am J Prev Med. 1999;17:198-202. CrossRef

(C) 2021 Advocate Aurora Health, Inc. 